\title{
P04-I4. Sequential HIV-I isolates from infected individuals reveals emergence of neutralization sensitive HIV-I strains in the course of infection
}

\author{
PN Nyambi*1, S Burda1 , C Williams¹, L Heyndrickx², G Vanham² and \\ MK Gorny ${ }^{1}$
}

\author{
Address: ${ }^{1}$ Pathology, New York University School of Medicine and V.A. Medical Center, New York, USA and ${ }^{2}$ Institute of Tropical Medicine, \\ Antwerp, Belgium \\ * Corresponding author
}

from AIDS Vaccine 2009

Paris, France. 19-22 October 2009

Published: 22 October 2009

Retrovirology 2009, 6(Suppl 3):P42 doi:I0.1 186/1742-4690-6-S3-P42

This abstract is available from: http://www.retrovirology.com/content/6/S3/P42

(c) 2009 Nyambi et al; licensee BioMed Central Ltd.

\section{Background}

Antibodies induced by a vaccine that will protect against infection by HIV-1 will be heterologous, yet, little is known on how virus evolution affects neutralization sensitivity to heterologous antibodies. Therefore, experiments were performed to test the sensitivity of sequential HIV-1 primary isolates to anti-HIV-1 monoclonal antibodies (mAbs) and to analyze changes in the neutralization epitopes.

\section{Methods}

Anti-HIV-1 mAbs directed at epitopes in the gp120 envelope (anti-V2, anti-V3, anti-CD4bd, and anti-carbohydrate) were tested in the TZM-bl neutralization assay with sequential primary HIV-1 isolates obtained from four HIV-1 infected drug naive individuals. The gp120 sequences from the sequential viruses were analyzed to identify changes in the neutralization sensitive epitopes.

\section{Results}

Overall, primary viruses collected from patients at first visit were all resistant to neutralization by anti-HIV-1 mAbs. Three of the four patients' sequential viruses evolved with increased sensitivity to neutralization by anti-V3 mAbs. In particular, virus collected from a patient 31 months after the first visit became more sensitive to neutralization by anti-V2, anti-V3, and anti-CD4bd mAbs. Another patient's later time point virus (collected after 11 months) evolved with increased sensitivity to five of six anti-V3 mAbs. The third patient's later time point virus (collected after 36 months) exhibited increased, but weak sensitivity to neutralization by anti-V3 mAbs. Anti-V3 mAbs exhibited the broadest and most potent neutralizing capacity to the evolving viruses over time. Sequence analysis of the envelope regions revealed amino acid changes occurring mainly outside of the mAb core epitope. Only few or no changes were noted in the V3 loop sequences.

\section{Conclusion}

This study demonstrates that HIV-1 viruses evolve with changes that increase neutralization sensitivity to some mAbs and that these changes occur outside the core neutralization sensitive epitope. Changes that cause increase in neutralization sensitivity may be useful in designing vaccines that may induce potent protective antibodies. 